\title{
Energetics and electronic structures of $\mathrm{N}$-doped graphene nanoribbons with pyridinic and graphitic edges
}

\author{
Airi Yasuma, Susumu Okada, Yanlin Gao, and Mina Maruyama* \\ Graduate School of Pure and Applied Sciences, University of Tsukuba, 1-1-1 Tennodai, Tsukuba \\ 305-8571, Japan
}

\begin{abstract}
Using the density functional theory, we study the energetics and electronic structures of graphene nanoribbons of which edges are substituted by $\mathrm{N}$ atoms. Our calculations showed that the edge $\mathrm{N}$ atoms with pyridinic structure mimic the $\pi$ environment of $C$ atoms, participating the $\pi$ electron network of $C$ atoms. In contrast, $\mathrm{N}$ atoms act as impurities for the $\pi$ electron network of the nanoribbons when their edges are terminated by $\mathrm{H}$ atoms, leading to versatile edge localized state those are hardly synthesized using $\mathrm{C}$ and $\mathrm{H}$ atoms. The total energy of $\mathrm{N}$-doped nanoribbons is the same as that of the pristine graphene nanoribbons, irrespective to the edge hydrogenation, and gradually decreases with increasing the nanoribbon width. We also found that the edge hydrogenation decrease and increase the stability of the $\mathrm{N}$-doped graphene nanoribbons with armchair and zigzag edges, respectively.
\end{abstract}

\section{Introduction}

A honeycomb covalent network of carbon atoms endows graphene with the semiconducting electronic structure with vanishing density of states at the Fermi level because of the conical dispersion band around the K points. ${ }^{1-3)}$ This electronic band structure leads to the unusual quantum Hall effect and results in remarkable carrier mobility of a few hundred thousand $\left.\mathrm{cm}^{2} / \mathrm{Vs}^{4},{ }^{4}\right)$ making graphene a unique and emerging material. Accordingly, graphene has been keeping a premier position in fundamental and applied sciences, ever since its discovery and synthesis. ${ }^{6,7)}$ In addition, graphene is known to be a starting material for designing other nanoscale materials with usual electronic structures by imposing appropriate boundary conditions. Carbon nanotubes are one of representative examples of such derivatives by imposing a tubular boundary condition onto graphene strips, being known to possess either metallic or semiconducting electronic properties depending on the atomic arrangements along their circumferences. ${ }^{8-10)}$ An open boundary condition, edges or pores on graphene derives, leads to further variation

${ }^{*}$ E-mail address: mmaruyama@comas.frsc.tsukuba.ac.jp 
in the electronic properties. It is well known that graphene nanoribbons possess peculiar electronic structures according to their width and edge atomic arrangements: ${ }^{11-15)}$ Graphene nanoribbons with zigzag edges possess edge localized states known as edge states arising from the topological condition around the edge atomic sites, while nanoribbons with armchair edges have either metallic or semiconducting properties depending on the discretized conditions imposed on graphene. Besides the edges, nanoscale pores introduced into graphene induce further variations in their electronic structures. ${ }^{16-18)}$

Borders between graphene and foreign materials cause peculiar electronic structure around the borders depending on their shapes and sizes. ${ }^{19-21)}$ A zigzag border between graphene and hexagonal boron nitride $(\mathrm{hBN})$ causes peculiar border localized states as the case of graphene nanoribbons with zigzag edges, because of the hybridization between edge states of graphene and hBN at the borders. ${ }^{19-21)}$ In addition to the bulk borders, substitutional doping of foreign atoms for graphene also induces rich variation in the electronic structure of atom-doped graphene, which is tuneable by selecting appropriate atom species and local network topology around the dopant sites. ${ }^{22)}$ According to the electronic structure variation, for instance, $\mathrm{N}$-doped graphene exhibits catalytic activity for the oxygen reduction reaction by controlling the proton or hydrogen concentration. ${ }^{23,24)}$ On the other hand, pyridine, known to be the smallest N-doped hydrocarbon, exhibits qualitatively the same electronic structure to that of benzene as far as $\pi$ electron states are concerned..$^{25-27)}$ These facts arouse a question whether the substitutionally doped $\mathrm{N}$ atoms into the edge atomic sites of graphene nanoribbons affect their characteristic electronic structures or not.

In this manuscript, we aim to investigate the energetics and electronic structures of graphene nanoribbons whose edge atomic sites are substituted by $\mathrm{N}$ atoms for providing the answers for the above question, using the density functional theory (DFT). Our calculations showed that both zigzag and armchair graphene nanoribbons with pyridinic $\mathrm{N}$ on their edges show qualitatively the same electronic structure near the Fermi level except the fully saturated $\sigma$ state of $\mathrm{N}$ atoms, indicating that the pyridinic $\mathrm{N}$ atom mimics the hydrogenated $\mathrm{C}$ atom as far as $\pi$ electron states are concerned. Hydrogenation of edge $\mathrm{N}$ atoms causes substantial electronic structure modulation, leading to another edge localized states for both zigzag and armchair edges. We also found that the edge hydrogenation decrease and increase the stability of the N-doped graphene nanoribbons with armchair and zigzag edges, respectively. 


\section{Calculation method and models}

In this work, we studied the geometric and electronic structures of N-doped graphene nanoribbons using $\mathrm{DFT}^{28,29)}$ implemented in a program package STATE. ${ }^{30)}$ We used the generalized gradient approximation with the Perdew-Burke-Ernzerhof functional ${ }^{31,32)}$ to describe the exchange-correlation potential energy among interacting electrons. Ultrasoft pseudopotentials generated by the Vanderbilt scheme were used to describe electron-ion interactions. ${ }^{33)}$ Valence wave functions and charge densities were expanded in terms of the plane wave basis set with cutoff energies of 25 and 225 Ry, respectively. The atomic structures were fully optimized until the force acting on each atom was less than $5 \mathrm{mRy} / \AA$ under the fixed lattice parameter along the nanoribbon of 4.26 and $2.46 \AA$ for armchair and zigzag nanoribbons, respectively. Integration over the onedimensional Brillouin zone was carried out using equidistant $k$-point sampling in which $8 k$-points were taken along nanoribbon direction, which give sufficient convergence for both geometric and electronic structures of graphene nanoribbons with arbitrarily edges. ${ }^{35)}$

In the present work, we consider $\mathrm{N}$-doped zigzag and armchair graphene nanoribbons with the nanoribbon width ranging from the narrowest to approximately $30 \AA$. For the armchair nanoribbons, the edge $\mathrm{C}$ sites are alternately substituted by $\mathrm{N}$ without and with $\mathrm{H}$ atom, leading to pyridinic and graphitic structures [Figs. 1(a) and (b)]. For the zigzag edges, all edge atomic sites are substituted by $\mathrm{N}$ atoms without and with $\mathrm{H}$ terminations [Figs. 1(c) and 1(d)]. To simulate an isolated N-doped graphene nanoribbons, each nanoribbon is separated by its periodic images by 6 - and $10-\AA$ vacuum regions normal and lateral directions of the nanoribbon, respectively.

\section{Results and discussion}

Figure 2 shows the total energy per heavy atom of N-doped graphene nanoribbons as a function of their width. The total energy $\varepsilon$ is evaluated by

$$
\varepsilon=\frac{E_{\mathrm{NR}}-N_{\mathrm{C}} \mu_{\mathrm{C}}-N_{\mathrm{N}} \mu_{\mathrm{N}}-N_{\mathrm{H}} \mu_{\mathrm{H}}}{N_{\mathrm{C}}+N_{\mathrm{N}}}
$$

where $E_{\mathrm{NR}}, \mu_{\mathrm{C}}, \mu_{\mathrm{N}}, \mu_{\mathrm{H}}, N_{\mathrm{C}}, N_{\mathrm{N}}$, and $N_{\mathrm{H}}$ indicate the total energy of nanoribbons, the chemical potential of $\mathrm{C}$ evaluated by graphene, the chemical potential of $\mathrm{N}$ evaluated $\mathrm{N}_{2}$ molecule, the chemical potential of $\mathrm{H}$, the number of $\mathrm{C}$, the number of $\mathrm{N}$, and the number of $\mathrm{H}$, respectively. The total energies of all nanoribbons gradually decrease with increasing their width. We also find that the total energy of the N-doped graphene 
(a)

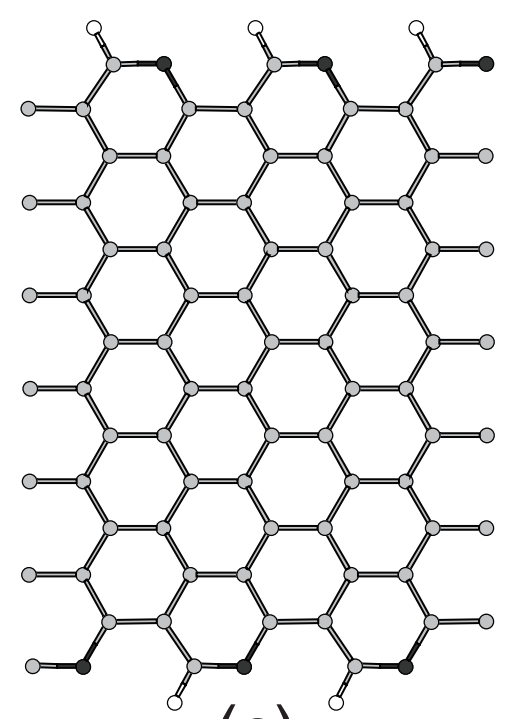

(c)

(b)

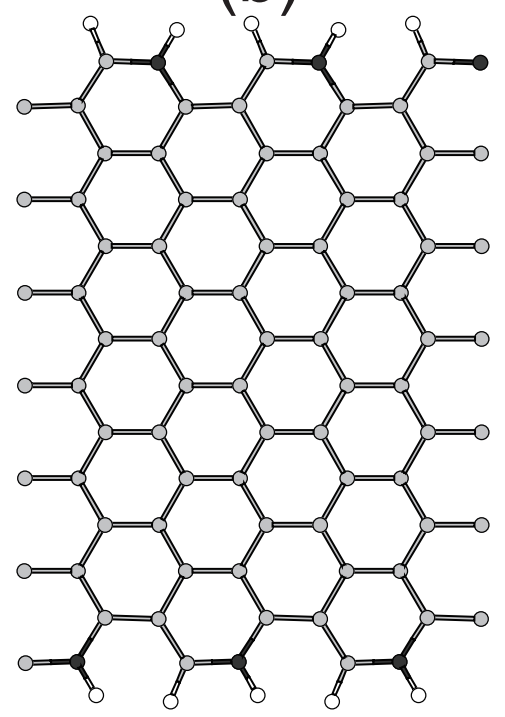

(d)

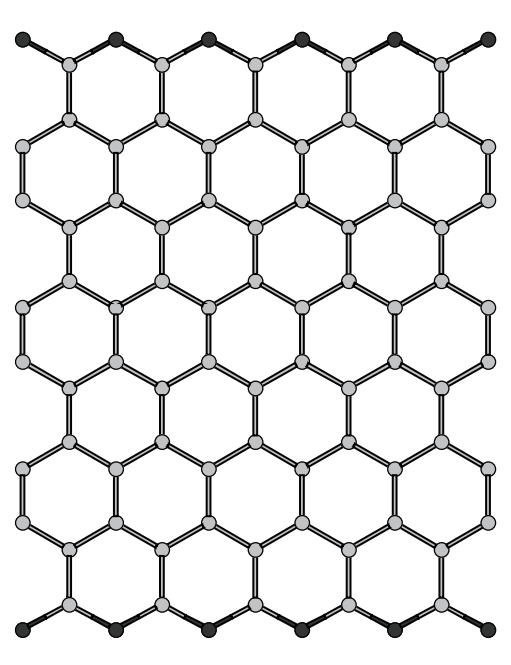

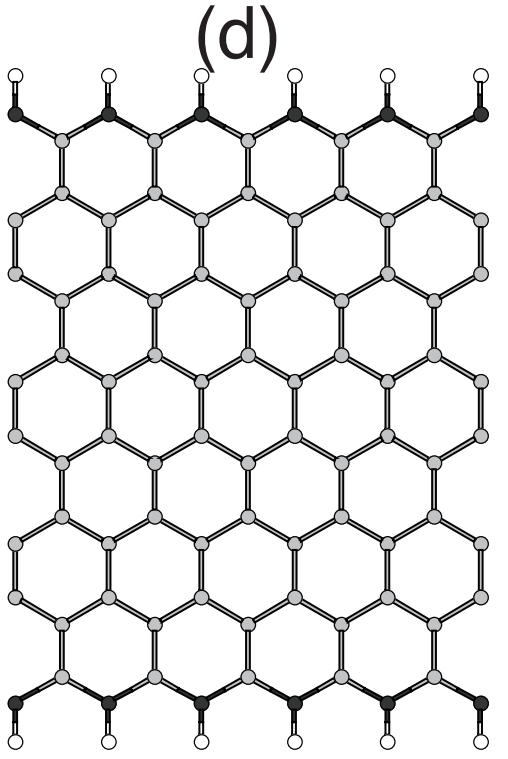

Fig. 1. Structural models of N-doped graphene nanoribbons with (a) the pyridinic armchair, (b) the graphitic armchair, (c) the pyridinic zigzag, and (d) the graphitic zigzag edges. Gray, black, and white circles denote $\mathrm{C}, \mathrm{N}$, and $\mathrm{H}$ atoms, respectively. Ribbon directions correspond with the horizontal direction in each figure.

nanoribbons is slightly higher than that of the hydrogenated graphene nanoribbons by about a few to a few tens meV per atoms, indicating that the N-doped graphene nanoribbons are substantially stable. For the armchair nanoribbons, the edge hydrogenation causes the increase of the total energy by a few tens meV per atom, indicating that the hydrogenation destabilizes the nanoribbons. In contrast, the total energy of the $\mathrm{N}$-doped nanoribbons with zigzag edges decrease by about a few tens meV per atom 


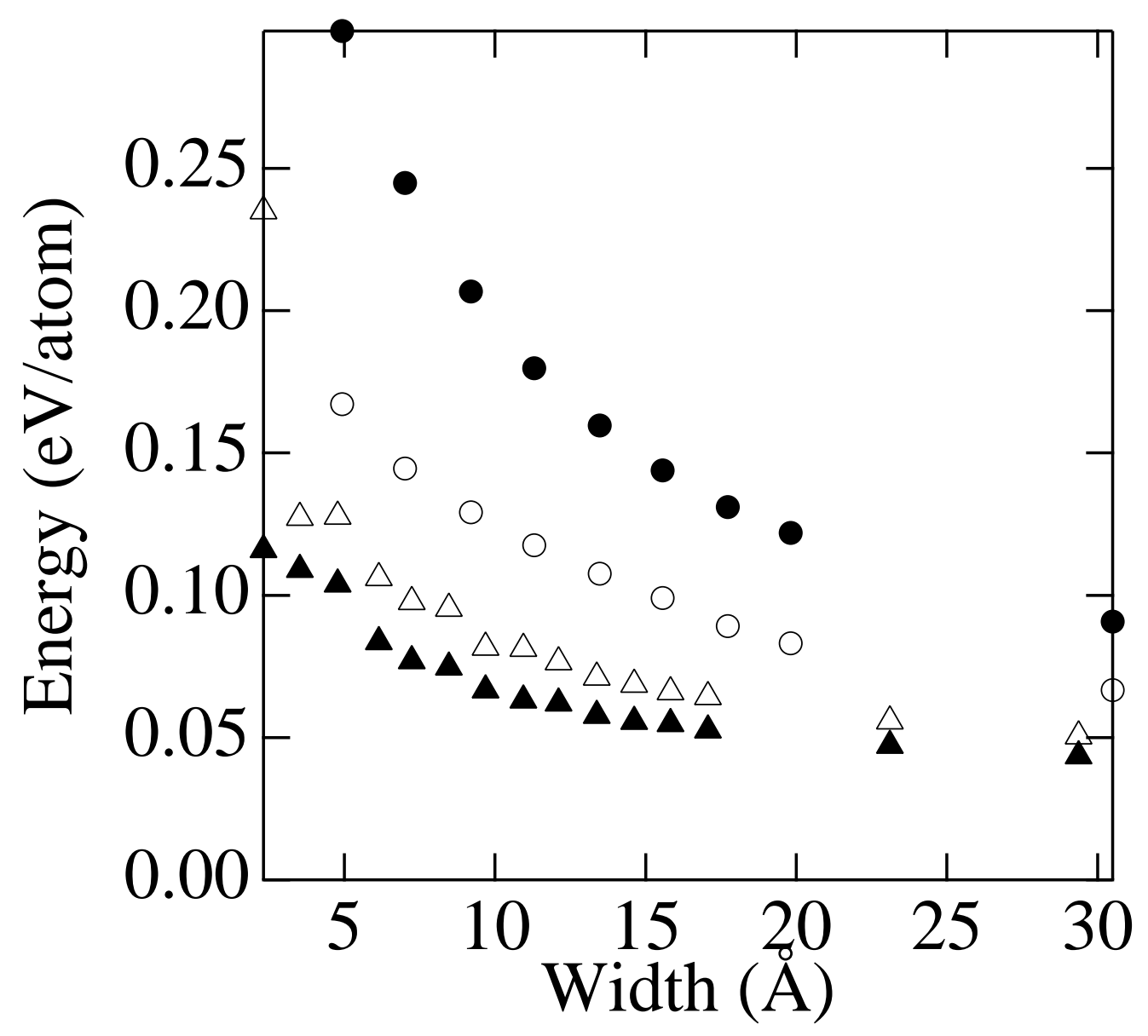

Fig. 2. Total energy per atom of N-doped graphene nanoribbons as a function of their width. Triangles and circles denote the N-doped armchair and N-doped zigzag nanoribbons, respectively. Filled and open marks correspond with the pyridinic (clean) and graphitic (hydrogenated) N edges, respectively.

by attaching $\mathrm{H}$ atoms to edge $\mathrm{N}$ atomic site, as the case of pristine graphene nanoribbons. It should be noted that the energy difference between pyridinic and graphitic (hydrogenated) edges are about a few tens meV irrespective of the edge shapes, which is remarkably smaller than that for the pristine graphene edges with and without hydrogen.

Figure 3(a) shows the electronic energy band of N-doped graphene nanoribbons with $29.36 \AA$ width and pyridinic armchair edges. For the armchair nanoribbon with pyridinic edges, the nanoribbon are semiconductor with direct band gap at the $\Gamma$ point. The electronic states around the gap is qualitatively the same as those of the pristine 
(a)

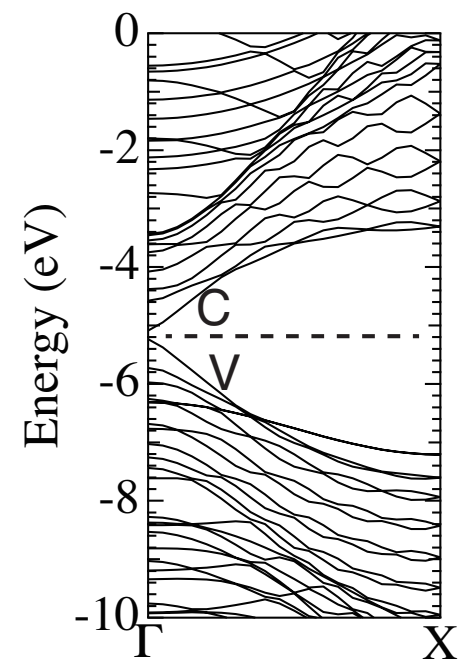

(c)

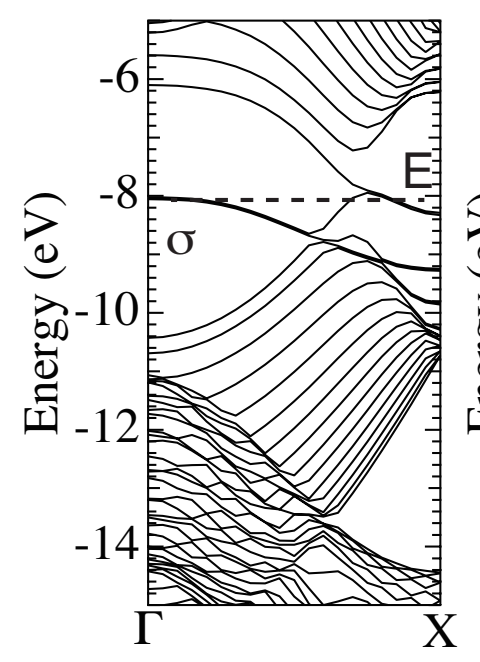

(b)

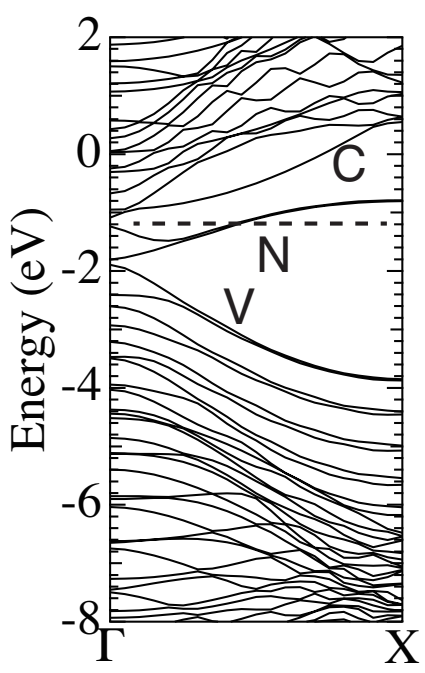

(d)

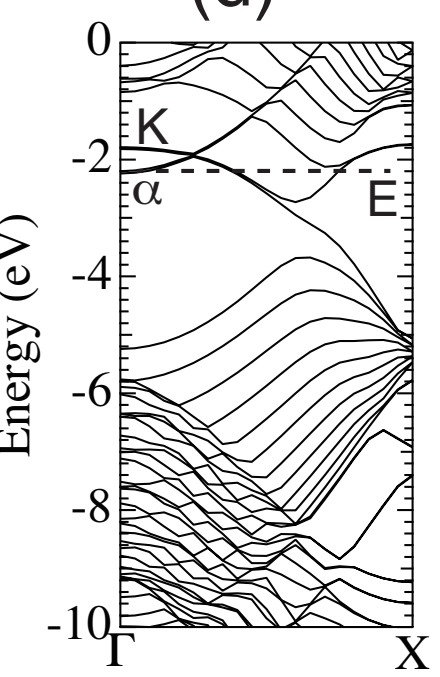

Fig. 3. Electronic energy band of N-doped graphene nanoribbons with (a) the pyridinic armchair, (b) the graphitic armchair, (c) the pyridinic zigzag, and (d) the graphitic zigzag edges. These nanoribbons have their width of 29.36 and $30.49 \AA$ for armchair and zigzag edges, respectively. The energy is measured from the that of the vacuum level. The horizontal dotted line denotes the Fermi level energy.

graphene nanoribbons with hydrogenated armchair edges, even though the one of two edge atomic sites is substituted by $\mathrm{N}$ atom. The states $1 \mathrm{eV}$ below the valence band top has a relatively small dispersion owing to the $\sigma$ states nature at $\mathrm{N}$ atoms. Furthermore, the band gap of the armchair nanoribbons with the pyridinic $\mathrm{N}$ edges also depends on their width as the case of the pristine graphene nanoribbons: The fundamental energy 


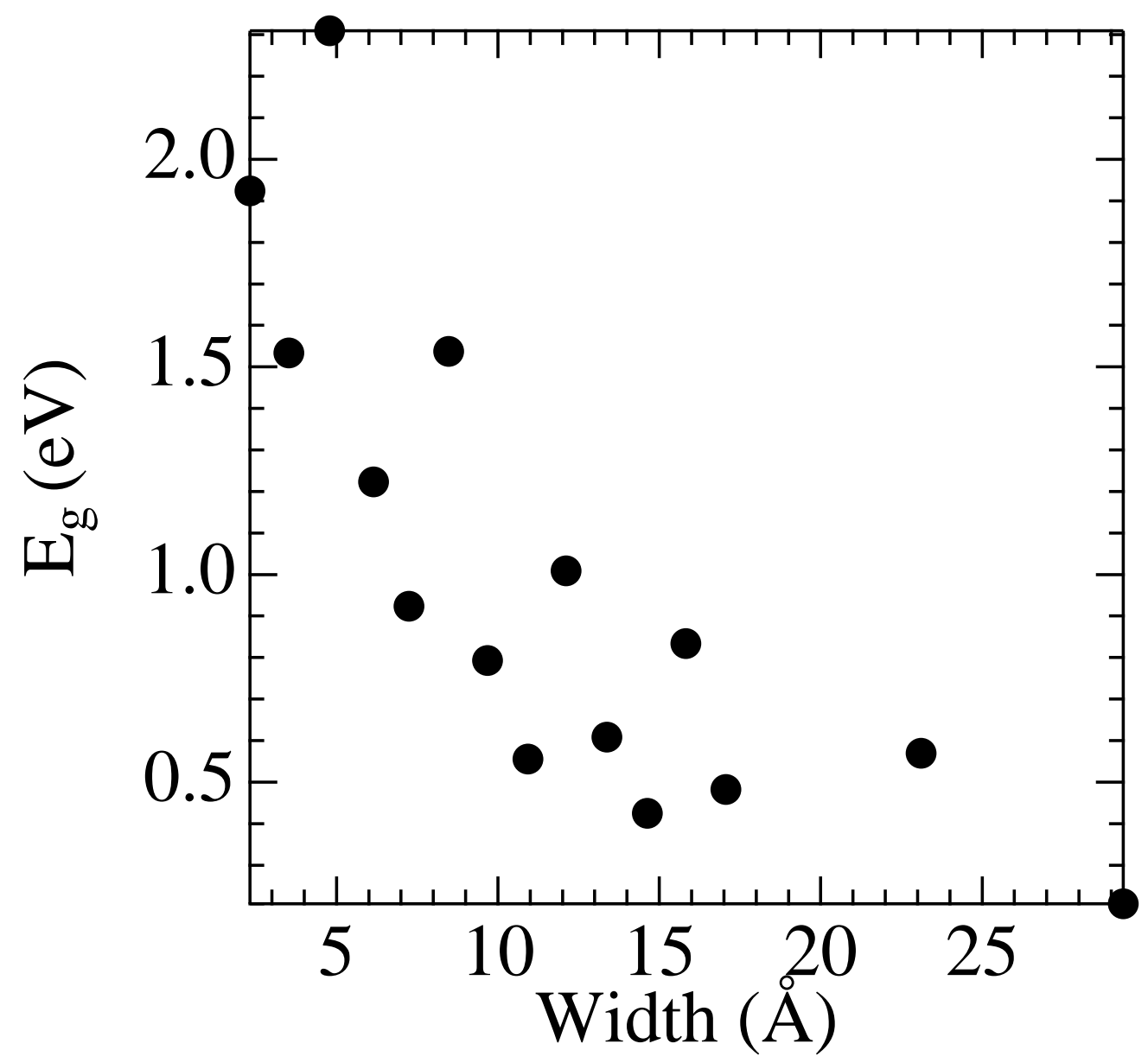

Fig. 4. The band gap of the N-doped graphene with the pyridinic armchair edges as a function of their width.

gap of these nanoribbons varies in accordance with a triple periodicity and asymptotically approaches zero with increase of their width (Fig. 4). The characteristic feature and the band gap is the same as that of the pristine nanoribbons with hydrogenated armchair edges, indicating that the $\mathrm{N}$ atoms substituting one of two edge $\mathrm{C}$ atoms do not affect $\pi$ electron states of graphene nanoribbons. Therefore, $\mathrm{N}$ atoms with pyridinic structure provide the same electrostatic environment to $\pi$ electrons.

The edge hydrogenation on the pyridinic armchair edges causes substantial modulation in the electronic energy band [Fig. 3(b)]. Two less dispersive bands emerge in the gap and cross the Fermi level leading to the metallic nature. Interestingly, these two bands are split into upper and lower branches around the $\Gamma$ point while they are degenerated each other for the rest of the wave numbers. This result indicates that the 
graphitic $\mathrm{N}$ atoms at the edges do not mimic $\mathrm{C}$ atoms, but that they act as impurities for $\mathrm{C}$ atomic networks causing the Klein type armchair edge for $\pi$ electron networks. In a previous theoretical work, the armchair nanoribbons with the Klein edges causes the half-filled flat dispersion band at the Fermi level, owing to the broken symmetry around the edge atomic sites. ${ }^{14)}$ Therefore, graphitic $\mathrm{N}$ atoms at the armchair edges effectively causes the Klein edges for $\pi$ electrons on the armchair graphene nanoribbons.

The N-doped graphene nanoribbon with $30.49 \AA$ width and the pyridinic zigzag edges also exhibits almost the same electronic structure as that of the pristine nanoribbons with zigzag $\mathrm{C}$ edges, except the less dispersive band just below the Fermi level arising from the $\sigma$ states of $\mathrm{N}$ atoms [Fig. 3(c)]. The doubly degenerated flat dispersion band emerges around the zone boundary, and they are smoothly connected to the bulk dispersive $\pi$ electron states as the usual edge states in the pristine zigzag graphene nanoribbons. Thus, the pyridinic $\mathrm{N}$ atoms at the zigzag edges also do not affect the $\pi$ electrons on the graphitic hexagonal networks.

As the case of the armchair edges, the hydrogenation of edge $\mathrm{N}$ atoms substantially modulates the electronic structure near the Fermi level [Fig. 3(d)]: Doubly degenerated flat bands emerge at and around the $\Gamma$ point, instead of the edge state around the zone boundary. The characteristic band dispersion of these states is the same as that of the Klein's edge states of the graphene nanoribbons with the Klein edges. ${ }^{15)}$ In addition to the flat dispersion bands around the $\Gamma$ point, doubly degenerated electron states with quadratic dispersion emerge and cross the Femi level at the $\Gamma$ point, indicating that their nearly free electron states nature. Thus, in the case of the zigzag edges with graphitic $\mathrm{N}$ atoms, $\pi$ electron systems are terminated at the $\mathrm{C}$ atoms adjacent to the $\mathrm{N}$ atoms.

To give the theoretical insight into the energetics and electronic structures of Ndoped graphene nanoribbons with pyridinic and graphitic edges, we investigate squared wave function near the Fermi level of N-doped graphene nanoribbons (Fig. 5). The wave functions of the valence band top and the conduction band bottom of the armchair nanoribbon with the pyridinic edges are extended throughout the nanoribbons with $\pi$ state nature as the case of the pristine graphene nanoribbon, even though it contains $\mathrm{N}$ atom at its edges [Fig. 5(a)]. But, it should be noted that chemical difference of $\mathrm{N}$ to $\mathrm{C}$ atoms make the $\pi$ state asymmetric distribution.

The edge hydrogenation of pyridinic armchair edges causes substantial modulation not only in the electronic energy band but also in the wave function distribution [Fig. 
(a)
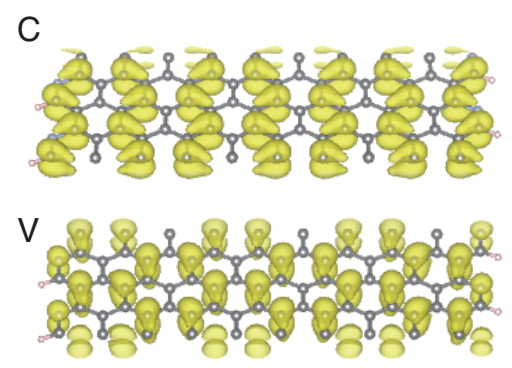

(c)

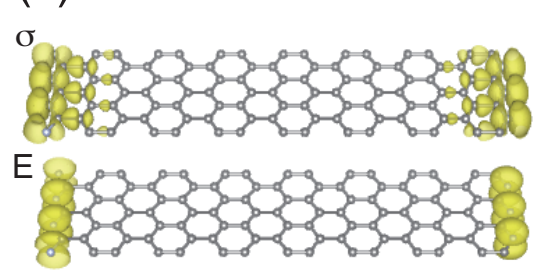

(b)
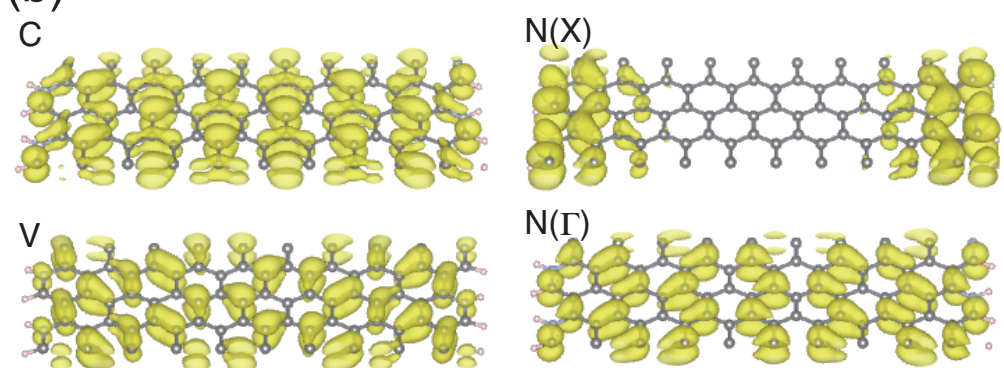

(d)

$\mathrm{K}$

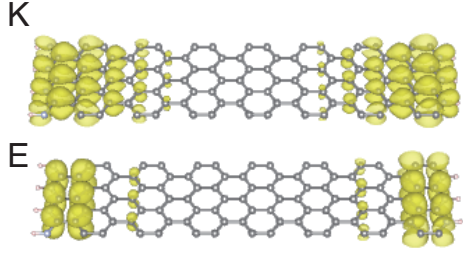

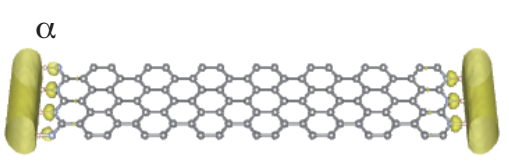

Fig. 5. (Color online) Isosurfaces of squared wave function of N-doped graphene nanoribbons with (a) the pyridinic armchair, (b) the graphitic armchair, (c) the pyridinic zigzag, and (d) the graphitic zigzag edges. These nanoribbons have their width of 29.36 and $30.49 \AA$ for armchair and zigzag edges, respectively. Labels in each figure correspond the band indexes in Fig. 3. Gray, cyan, and pink balls indicate $\mathrm{C}, \mathrm{N}$, and $\mathrm{H}$ atoms, respectively.

5(b)]: Dispersive valence and conduction bands labeled V and C, respectively, exhibit extended $\pi$ electron states nature, but their distribution slightly modulated from those of the $\mathrm{V}$ and $\mathrm{C}$ stats in the pyridinic armchair edges. For the flat band labeled $\mathrm{N}$, the state exhibits unusual distribution nature: The states are localized around the edge atomic site at the $\mathrm{X}$ point with their penetration length of $7.5 \AA$, although the states are extended throughout the nanoribbons with conventional $\pi$ electron nature at the $\Gamma$ point. Note that the localized nature of these states gradually decreases with approaching the $\Gamma$ point. The localized nature and flat band of these states are induced by the sublattice imbalance around the edge atomic sites, so that the states could be classified the extended version of the edge states. The formation of the edge localized states at the Fermi level increases the total energy $\varepsilon$ of the N-doped graphene nanoribbons with graphitic or hydrogenated armchair edges.

For the case of the nanoribbon with pyridinic zigzag edges [Fig, 5(e)], we found that the flat band around the $\mathrm{X}$ point has the edge state nature: The sate is completely localized on the edge $\mathrm{N}$ atoms with nonbonding $\pi$ nature. Furthermore, the state gradually penetrates inner $\mathrm{C}$ region with decreasing the wave number. Therefore, we conclude that the $\mathrm{p}_{z}$ orbital of pyridinic $\mathrm{N}$ atom qualitatively mimics $\pi$ state of $\mathrm{C}$ atom, because 
the edge states retains its characteristic feature in the nanoribbons with pyridinic edges.

By attaching $\mathrm{H}$ atoms to the $\mathrm{N}$ atoms, the edge states around the $\mathrm{X}$ point disappear in the band structure. In the case, we found a pair of small dispersion bands at and around the $\Gamma$ point. The state is distributed not only on edge $\mathrm{N}$ atoms but also on the $\mathrm{C}$ atoms around the border with $\mathrm{N}$ atoms. Also the states gradually decrease its amplitude with approaching inner region of the nanoribbon, still exhibiting their edge localized nature [Fig. 5(d)]. The longer penetration depth of the state even at the $\Gamma$ point indicates that the small dispersion band at the Fermi level is ascribed to the Klein's edge states. Therefore, the hydrogenation of $\mathrm{N}$ atom effectively renders the $\mathrm{N}$-doped zigzag nanoribbons the graphene nanoribbons with the Klein edges. In addition, the hydrogenation also causes substantial downward shift of the nearly free electron states alongside the nanoribbon edges owing to the strong dipole moment of $\mathrm{N}-\mathrm{H}$ bond. ${ }^{36-42)}$ Therefore, the N-doped nanoribbons with graphitic zigzag edges may exhibit unusual transport properties attributed from the free electron alongside the nanoribbon and the relatively heavy electron around the edge atomic sites.

\section{Summary}

In this manuscript, we investigated the energetics and electronic structures of graphene nanoribbons whose edge atomic sites are substituted by $\mathrm{N}$ atoms using the DFT with GGA. Our calculations showed that the both zigzag and armchair graphene nanoribbons with pyridinic $\mathrm{N}$ on their edges show the qualitatively the same electronic structure to that of the graphene nanoribbons near the Fermi level except the fully saturated $\sigma$ state of $\mathrm{N}$ atoms, indicating that the pyridinic $\mathrm{N}$ atoms mimic hydrogenated $\mathrm{C}$ atoms as far as $\pi$ electron states are concerned. Hydrogenation of edge $\mathrm{N}$ atoms causes substantial electronic structure modulation, leading to another edge localized state for both zigzag and armchair edges. For armchair and zigzag edges, hydrogenated $\mathrm{N}$ atoms at the edge act as impurity in $\pi$ electron networks of nanoribbons and cause interfaces with graphene region where the Klein type edges for $\pi$ electron systems are formed. The total energy of the nanoribbon gradually decreases with increasing the nanoribbon width as the case of that of the pristine graphene nanoribbons. We also found that the edge hydrogenation decreases and increases the stability of the N-doped graphene nanoribbons with armchair and zigzag edges, respectively, owing to the substantial modulation of electronic structures of N-doped graphene nanoribbons at the Fermi level. 


\section{Acknowledgments}

The author thanks JST-CREST Grant Numbers JPMJCR1532 and JPMJCR1715 from the Japan Science and Technology Agency, JSPS KAKENHI Grant Numbers JP17H01069, JP16H00898, and JP16H06331 from the Japan Society for the Promotion of Science, and the Joint Research Program on Zero-Emission Energy Research, Institute of Advanced Energy, Kyoto University. Part of the calculations was performed on an NEC SX-Ace at the Cybermedia Center at Osaka University and on an SGI ICE $\mathrm{XA} / \mathrm{UV}$ at the Institute of Solid State Physics, The University of Tokyo. 


\section{References}

1) G. S. Painter and D. E. Ellis, Phys. Rev. 1, 4747 (1970).

2) F. Bassani and G. P. Parravicini, Nuovo Cimento B 50, 95 (1967).

3) M. Posternak, A. Baldereschi, A. J. Freeman, E. Wimmer, and M. Weinert, Phys. Rev. Lett. 50, 761 (1983).

4) K. S. Novoselov, A. K. Geim, S. V. Morozov, D. Jiang, M. I. Katsnelson, I. V. Grigorieva, S. V. Dubonos, and A. A. Firsov, Nature, 438, 197 (2005).

5) Y. Zhang, Y.-W. Tan, H. L. Stormer, and P. Kim, Nature, 438, 201 (2005).

6) M. S. Dresselhaus and G. Dresselhaus: Adv. Phys. 30, 139 (1981).

7) A. H. Castro Neto, F. Guinea, N. M. R. Peres, K. S. Novoselov and A. K. Geim: Rev. Mod. Phys. 81, 109 (2009).

8) N. Hamada, S.-I. Sawada, and A. Oshiyama, Phys. Rev. Lett. 68, 1579 (1992).

9) R. Saito, M. Fujita, M.S. Dresselhaus, and G. Dresselhaus, Appl. Phys. Lett. 60, 2204 (1992).

10) K. Tanaka, K. Okahara, M. Okada, and T. Yamabe, Chem. Phys. Lett. 191, 469 (1992).

11) M. Fujita, K. Wakabayashi, K. Nakada, K. Kusakabe, J. Phys. Soc. Jpn. 65, 1920 (1996).

12) K. Nakada, M. Fujita, G. Dresselhaus, and M.S. Dresselhaus, Phys. Rev. B 54, 17954 (1996).

13) Y. Miyamoto, K. Nakada, and M. Fujita, Phys. Rev. B 59, 9858 (1999).

14) K. Wakabayashi, S. Okada, R. TOmita, S. Fujimoto, and Y. Natsume, J. Phys. Soc. Jpn. 79, 034706 (2010).

15) D. J. Klein, Chem. Phys. Lett. 217, 261 (1994).

16) N. Shima and H. Aoki, Phys. Rev. Lett. 71, 4389 (1993).

17) M. Maruyama, N. T. Cuong, and S. Okada, Carbon 109, 755 (2016).

18) M. Maruyama and S. Okada, Carbon 125, 530 (2017).

19) S. Okada, M. Igami, K. Nakada, and A. Oshiyama, Phys. Rev. B 62, 9896 (2000).

20) S. Okada and A. Oshiyama, Phys. Rev. Lett. 87, 146803 (2001).

21) M. Maruyama and S. Okada, J. Phys. Chem. C 120, 1293 (2016).

22) Y. Fujimoto and S. Saito, Phys. Rev. B 84, 245446 (2011).

23) T. Kondo, T. Suzuki, and J. Nakamura, J. Phys. Chem. Lett. 2, 577 (2011).

24) T. Kondo, S. Casolo, T. Suzuki, T. Shikano, M. Sakurai, Y. Harada, M. Saito, M. 
Oshima, M. I. Trioni, G. F. Tantardini, and J. Nakamura, Phys. Rev. B 86, 035436 (2012)

25) J. D. Bene and H. H. Jaffé, J. Chem. Phys. 48, 1807 (1968).

26) J. Spanget-Larsen, J. Electron Spectros. Relat. Phenomena 2, 33 (1973).

27) D. Chen, S.-J. Su, and Y. Cao, J. Mater. Chem. C 2, 9565 (2014).

28) P. Hohenberg and W. Kohn, Phys. Rev. 136, B864 (1964).

29) W. Kohn and L. J. Sham, Phys. Rev. 140, A1144 (1965).

30) Y. Morikawa, K. Iwata, and K. Terakura, Appl. Surf. Sci. 169-170, 11 (2001).

31) J. P. Perdew, K. Burke, and M. Ernzerhof, Phys. Rev. Lett. 77, 3865 (1997).

32) J. P. Perdew, K. Burke, and M. Ernzerhof, Phys. Rev. Lett. 78, 1396 (1997).

33) D. Vanderbilt, Phys. Rev. B 41, 7892 (1990).

34) A. Yamanaka and S. Okada, Carbon 96, 351 (2016).

35) M. Posternak, A. Baldereschi, A. J. Freeman, E. Wimmer, and M. Weinert, Phys. Rev. Lett. 50, 761 (1983).

36) M. Posternak, A. Baldereschi, A. J. Freeman, and E. Wimmer, Phys. Rev. Lett. 52, 863 (1984).

37) A. Catellani, M. Posternak, A. Baldereschi, and A. J. Freeman, Phys. Rev. B 36, 6105 (1987).

38) X. Blase, A. Rubio, S. G. Louie, and M. L. Cohen, Phys. Rev. B 51, 6868 (1995).

39) Q. Liu, Z. Li, and J. Yang, Chinese J. Chem. Phys. 24, 22 (2011).

40) A. Yamanaka and S. Okada, Appl. Phys. Express 7, 125103 (2014).

41) A. Yamanaka and S. Okada, Sci. Rep. 6, 30653 (2016). 